\title{
HACIA UNA HISTORIA \\ DE LA INTERDISCIPLINARIEDAD \\ EN LA ARQUEOLOGÍA ESPAÑOLA: INTRODUCIENDO UNA NUEVA PERSPECTIVA*
}

\section{TOWARDS A HISTORY OF INTERDISCIPLINARITY IN SPANISH ARCHAEOLOGY: INTRODUCING A NEW PERSPECTIVE}

\author{
Margarita DíAZ-ANDreu** \\ Profesora de Investigación de ICREA. Institució Catalana de Recerca i Estudis Avançats (ICREA). \\ Departament d'Història i Arqueologia, Universitat de Barcelona \\ Laura Coltofean \\ Departament d'Historia i Arqueologia, Universitat de Barcelona
}

\begin{abstract}
RESUMEN. En este trabajo que introduce el dosier «Hacia una historia de la interdisciplinariedad en Arqueología», coordinado por las mismas autoras, se define, en primer lugar, qué se entiende por interdisciplinariedad y otros conceptos afines como pluridisciplinariedad. Se realiza entonces un repaso diacrónico de cómo las relaciones interdisciplinares se han producido en la arqueología espańola desde el siglo xix hasta la situación actual. Esta síntesis revela un cierto retraso inicial con respecto a otros países, pese a que desde el inicio sí que se encuentren especialistas en otras ramas del saber que aportan con su conocimiento especializado a la arqueología. Sin embargo, solo será en el siglo xx cuando llegue a aparecer una interdisciplinariedad estrictamente hablando, mientras que la progresiva aceptación de la valía de las contribuciones de otras ciencias lleva ya en estas últimas décadas a un énfasis no tanto de la interdisciplinariedad sino de la pluridisciplinariedad dentro de la propia arqueología en Espańa.
\end{abstract}

PALABRAS CLAVE: Interdisciplinariedad, pluridisciplinariedad, historia de la Arqueología, Espańa, Ciencias Arqueológicas.

ABSTRACT. This introductory article to the «Towards a History of Interdisciplinarity in Archaeology» dossier, coordinated by the authors, starts with a definition of interdisciplinarity and other related concepts such as pluridisciplinarity. A diachronic review is then undertaken looking at how interdisciplinary relations have taken place in Spanish archaeology from the nineteenth century until today. This synthetic overview reveals a certain initial delay with respect to other countries. From early days of the discipline, a few specialists in other branches of knowledge contributed with their specialized knowledge to archaeology, although strictly speaking interdisciplinarity only took place in the twentieth century. The progressive acceptance of the value of the contributions of other sciences has led in recent decades to an emphasis not so much on interdisciplinarity but on pluridisciplinarity within Spanish archaeology.

KEYWORDS: Interdisciplinarity, pluridisciplinarity, history of Archaeology, Spain, archaeological sciences.

* Este trabajo se enmarca en el proyecto de investigación HAR2016-80271-P, financiado por el MINECO-Ministerio de Economía y Competitividad cuya Investigadora Principal es Margarita Díaz-Andreu, subvencionado por la Agencia Estatal de Investigación (AEI) y el Fondo Europeo para el Desarrollo Regional (FEDER).

\footnotetext{
** Correspondencia a / Correspondence to: Margarita Díaz-Andreu, Universitat de Barcelona - m.diaz-andreu@ub.edu - https://orcid.org/00000003-1043-2336.

Cómo citar / How to cite: Díaz-Andreu, Margarita; Coltofean, Laura (2020), "Hacia una historia de la interdisciplinariedad en la Arqueología española: introduciendo una nueva perspectiva», Veleia, 37, 13-32. (https://doi.org/10.1387/veleia.21063).
}

Recibido: 22 septiembre 2019; aceptado: 29 septiembre 2019.

ISSN 0213-2095 - elSSN 2444-3565 / (C) 2020 UPV/EHU 
El dosier «Hacia una historia de la interdisciplinariedad en Arqueología» nace de una reflexión colectiva realizada en las distintas reuniones llevadas a cabo por los miembros del proyecto "Arqueología e interdisciplinariedad: una investigación arqueológico-histórica sobre las relaciones interdisciplinares en la Historia de la Arqueología (siglos XIX y Xx)» (proyecto HAR2016-80271-P, financiado por el Ministerio de Economía y Competitividad (www.interarqweb.wordpress.com), y en especial por el seminario realizado el 15 de junio de 2019. El objetivo del proyecto, así como del seminario, ha sido el analizar las relaciones de carácter interdisciplinar entre la Arqueología y otras ramas del saber a lo largo de los dos últimos siglos. En este compendio se recogen por tanto una serie de trabajos que ponderan sobre cómo la práctica interdisciplinar ha influido en la evolución y desarrollo de la arqueología en España. Como forma de contextualizar las distintas contribuciones en esta introducción, tras definir qué se entiende por interdisciplinariedad, pluridisciplinariedad y otros conceptos afines, realizamos una síntesis histórica en la que aludiremos a los distintos trabajos de los autores del dosier en los lugares oportunos.

Nuestra narración ha señalado que existió un cierto retraso en las relaciones interdisciplinares en un sentido estricto con respecto a otros países, aunque sensu lato estuvieron allí desde el principio. Con ello queremos apuntar que desde los inicios de los estudios arqueológicos aparecen individuos con conocimientos en otras ramas de la ciencia que pudieron ofrecer interpretaciones del pasado novedosas, precisamente gracias a su formación y práctica profesional no relacionada con las antigüedades, pero sin que, sin embargo, esto llevara al traspaso de conocimientos entre unos y otros especialistas. En la gran mayoría de los casos esta ausencia de transferencia se debió fundamentalmente a la poca profesionalización del saber arqueológico, tanto porque el enfoque de los profesionales fuera limitado (en gran parte del XIX estos prestaron una atención mayoritaria a lo romano prácticamente ignorando los restos materiales sobre todo de época prehistórica), como porque el número de arqueólogos fuera demasiado bajo. Estos factores fueron desapareciendo a lo largo del siglo xx, siendo solo en el último cuarto de esta centuria cuando se produce una normalización de la interdisciplinariedad —o más bien de la pluridisciplinariedad— en arqueología. En realidad, lo hace de una manera muy concreta: en un contexto político en el que todo cambio con respecto a lo anterior se valora como positivo, aunque continúe una cierta colaboración con miembros de otras ciencias, lo que se prima en este último periodo es especializar a nivel postdoctoral a los futuros profesionales arqueólogos en las otras ramas del saber para entonces integrarles profesionalmente en los propios departamentos de arqueología.

En estos últimos años se ha concedido una gran importancia a la interdisciplinariedad. A nivel europeo ha cobrado un papel protagonista en los proyectos del Horizonte 2020, lo que ha repercutido en los planes estatales y locales. Sin embargo, como veremos en este dosier que introducimos aquí, en arqueología — así como en muchas otras ramas del saber- la interdisciplinariedad dista de ser algo novedoso, puesto que ha producido desde su propia incepción como disciplina, aunque únicamente haya experimentado un proceso de normalización en estos últimos años. En este artículo aludiremos a los conceptos básicos referidos a la interdisciplinariedad para, posteriormente, hacer un repaso diacrónico de las diferentes etapas por la que ha transcurrido la colaboración entre la arqueología y otras disciplinas. Nuestro bosquejo histórico se ceñirá a España, aunque haremos alusión a procesos que han tenido lugar en otros países. Comenzaremos en el siglo XIX para ralentizar su mirada en lo acontecido en la siguiente centuria y en la presente. 


\section{Conceptos}

Interdisciplinariedad implica la relación entre disciplinas y por ello comenzaremos definiendo lo que por estas últimas entendemos. Una disciplina es una rama del conocimiento, instrucción, aprendizaje, enseńanza o educación, un conjunto de saberes que se pueden aprender y enseńar para formar a un individuo para su futuro profesional. Como explican Choi y Pak, «una disciplina se mantiene unida por una epistemología compartida, es decir, por una serie de suposiciones sobre la naturaleza del conocimiento y formas aceptables de generarlo o acumularlo» (Choi \& Pak 2008, E42). Por su proximidad epistemológica, siguiendo la norma común en nuestros días, agrupan estos autores las disciplinas en tres grandes subsistemas de conocimiento: las ciencias naturales, las ciencias sociales y las humanidades, para proponer acertadamente que cuanto más dispares epistemológicamente hablando sean las disciplinas que se combinan, mayor probabilidad habrá de alcanzar mejores resultados referentes a una nueva visión de un problema o tema complejo (Choi \& Pak 2008, E42).

Pese a la validez de las propuestas de Choi y Pak creemos conveniente apuntar que un factor imprescindible a tener en cuenta y que va a ser analizado en este dosier es que las disciplinas no son algo objetivo e inamovible, puesto que tienen historia. Las disciplinas son acuerdos sociales a los que se ha llegado en alguna época del pasado y cuyo contenido viene siendo continuamente renegociado. Si, por poner un ejemplo, la filosofía es una disciplina antigua ya existente entre los griegos, los contenidos de esta no lo son, puesto que lo que se aprendía para ser un filósofo entonces, en el siglo XIX o ahora ha cambiado de forma importante a lo largo de los siglos. Por su parte hay otras disciplinas que son de corte moderno, como la sociología, cuyo nacimiento como concepto, data de 1822, surgiendo solo más tarde como estudio tipificado. Esto significa que las fronteras entre las disciplinas no representan sino convenios realizados en un momento del tiempo, que se van transformando con la práctica y cuyos límites en cuanto a los saberes que incluyen no son sino contingentes. La realidad histórica de las disciplinas como ramas del conocimiento puede complicar un estudio como en el que se realiza en este volumen sobre la interdisciplinariedad en arqueología, puesto que disciplinas que en una época eran la misma se subdividen en dos diferentes en un determinado momento (véase la división entre arte y arqueología (Díaz-Andreu 1995), pudiéndose producir también el proceso contrario (hasta cierto punto todas las arqueo-x —zoología, botánica, etc.— actuales).

El mismo concepto de arqueología tal y como lo empleamos hoy en día es, por supuesto, histórico y hace apenas unos diez o, como mucho, veinte años muy pocos lo hubieran entendido de la misma manera. En este dosier entendemos como arqueología la rama del saber que busca el conocimiento de las sociedades a través del análisis de su cultura material y de las huellas dejadas en el paisaje de sus costumbres inmateriales. Esta definición es de hoy y se enfrenta a la forma en la que este concepto se había comprendido en Espańa y en todo el mundo occidental hasta hace bien poco, cuando los cursos de Arqueología ceñían sus contenidos a lecciones de arte griego y romano o cuando un grupo diferente todavía debatía si el vocablo simplemente debía reflejar una técnica y no un conjunto de saberes históricos. Desde los años 2000 la aparición de grados universitarios denominados como tal (Gracia Alonso \& Fullola Pericot 2005; Querol 2005) ha parecido imponer la definición que aquí estamos apoyando.

Klein y Newell, dos de los principales teóricos en el campo de la educación en los 90, ofrecían una definición de interdisciplinariedad que, según comentaban, reflejaba un consenso emergente entre aquellos que la practicaban y con la que estamos todavía hoy de acuerdo:

Los estudios interdisciplinares pueden definirse como un proceso de responder a una pregunta, resolver un problema o abordar un tema que es demasiado amplio o complejo para ser tratado ade- 
cuadamente por una sola disciplina o profesión... [y] se basa en perspectivas disciplinarias e integra sus puntos de vista [para producir] una perspectiva más completa. (Klein \& Newell 1997: 393)

Acabaremos este apartado apuntando que es importante distinguir entre interdisciplinariedad y otros conceptos similares, en parte superpuestos, como son la multidisciplinariedad, la transdisciplinariedad y la pluridisciplinariedad. La multidisciplinariedad se refiere a la suma de ciencias sin que haya un intercambio de saberes entre ellas, lo que ocurre, por ejemplo, entre los autores de una enciclopedia. La transdisciplinariedad se refiere a un ideal de unión de todas las ciencias, algo de lo que no trataremos en este dosier y, por último, la pluridisciplinariedad es la integración de dos disciplinas en una (Alvargonzález 2011; Choi \& Pak 2006).

\section{LA COLABORACIÓN INTERDISCIPLINAR EN EL SIGLO XIX}

La definición de disciplina tal y como se ha especificado en el apartado anterior implica no solo conocimiento, sino también instrucción, aprendizaje, enseńanza o educación y esto concede a las universidades un papel fundamental. La primera cátedra de arqueología en el mundo se funda en Leiden en $1818^{1}$. El que la ocupaba, Caspar Reuvens (1793-1835), tenía responsabilidades también como director del Gabinete de Antigüedades de la universidad, que albergaba una rica colección de antigüedades romanas y griegas que él aumentaría con otras egipcias, púnicas y locales. Esto último lo conseguiría gracias, entre otras cosas, a su excavación del Foro de Adriano de Voorburg, a las afueras de La Haya (Halbertsma 2008). Otras cátedras seguirían a esta: Georg Zoëga (1755-1809) en Kiel, entonces parte de Dinamarca, desde 1802, Jean-François Champollion en 1831 en Paris, y Ludwig Ross (1806-1859) en 1834 en la Universidad de Atenas, por citar solo las primeras (DíazAndreu 2007). Todas ellas tenían en común el estar exclusivamente relacionadas con el estudio de las grandes civilizaciones, y el estudio de otros tipos de pasado menos grandiosos como es la época prehistórica solo serían integrados en la enseñanza en el siglo xx (Callmer et al. 2006). Lo cierto es que, volviendo al xix, el número de expertos en las universidades era muy limitado y la gran mayoría de especialistas se encontraban en los museos y, en mucha menor medida, en los nacientes servicios de arqueología estatales. El número total de profesionales, por tanto, era sumamente exiguo, mientras que el de los que se interesaban y practicaban la arqueología era mucho mayor.

Aplicar las definiciones actuales de interdisciplinariedad al siglo XIX, sin embargo, nos lleva a dificultades. Hoy en día denominamos como arqueólogo amateur o vocacional a todo aquel o aquella que se gana la vida gracias a su oficio en otra profesión y practica la arqueología sin título que le permita entrar en el grupo oficial de arqueólogos. En el siglo xix la situación era más compleja puesto que la enseńanza distaba mucho de estar reglada a un nivel semejante al actual, y entre los profesionales, aunque sí que se esperaba un título universitario, este no necesariamente debía ser lo que hoy en día consideraríamos como adecuado. La gran mayoría de los que se dedicaban a la arqueología se ganaban la vida en otros oficios, pero hoy les incluimos en las historias de la arqueología como arqueólogos. La gran diversidad de formaciones ayudaría que existieran una gran cantidad de formas de hacer y de análisis surgidos en otras disciplinas aplicándose en arqueología. ¿Podríamos llamar a esto interdisciplinariedad? Sí, si en la definición de interdisciplinariedad en-

${ }^{1}$ Existe un precedente del que poco sabemos, la cátedra de arqueología de Olof Verelius de la Universidad de Uppsala de 1662 (Jensen 2004, 64; Klindt-Jensen 1975, 26). 
tendemos por relación entre disciplinas la que se produce entre diferentes saberes. Si, por el contrario, esperamos que exista un intercambio de conocimiento y técnicas entre un profesional de una disciplina y un colega de otra, entonces no. En estos primeros momentos en los que el cuerpo profesional es tan exiguo, las barreras de lo que uno puede hacer o no independientemente de su profesión son más flexibles y la colaboración en sí puede no existir, debido a la falta de profesionales en una de las ciencias. Por otra parte, se puede también dar el caso de profesionales que, a pesar de que provengan de otro oficio, no pongan sus conocimientos en este al servicio de la Arqueología.

Ofrezcamos varios ejemplos de lo relatado en el párrafo anterior, que hemos extraído en su mayor parte del Diccionario Histórico de la Arqueología en España (siglos XV-XX) (Díaz-Andreu, Mora \& Cortadella 2009). Cuando el geólogo Casiano de Prado y Vallo comienza a publicar sobre paleolítico en los años 1850 no había nadie en ningún museo con material arqueológico ni, desde 1867, en el Museo Arqueológico Nacional, con quien pudiera plantearse una colaboración, puesto que en aquel momento, ni a partir de la fundación de la Escuela Superior de Diplomática en 1856, los arqueólogos oficiales mostraron ningún interés en integrar la prehistoria remota entre las épocas. Tras De Prado aparecen otros como Guillermo MacPherson, Rogelio de Inchaurrandieta o Santiago Moreno (Goberna 1986, 29). Lo mismo ocurre con el geólogo-paleontólogo Juan Vilanova y Piera (1821-1893) a partir de los años 1860. Además de los geólogos encontramos en esta mitad del siglo xIx a los ingenieros como el de caminos Eduardo Saavedra y Moragas (1829-1912), a quien ya le interesan épocas más recientes. En su primer trabajo sobre la via romana entre Uxama y Augustóbriga puso una serie de conocimientos de su profesión al servicio de los arqueólogos que por entonces eran poco dados a salir de su gabinete. También los procedimientos aprendidos en ingeniería de minas serviría a Louis Siret (1860-1934) para sistematizar la prehistoria del sureste (Ayarzaguena Sanz 2018; Díaz-Andreu, Mora, \& Cortadella 2009; Puche Riart 2002). Otro grupo de arqueólogos vocacionales fueron los médicos como Gregorio Chil y Naranjo (1831-1901), quien se interesaría por el origen de los aborígenes canarios, o los farmacéuticos como Pere Alsius i Torrent (1839-1915), el descubridor de la mandíbula neanderthal de Bañolas (Díaz-Andreu, Mora \& Cortadella 2009). Todos ellos provenían de ciencias que en principio tenían poco que ver con el estudio de las sociedades y quizá por ese alejamiento epistemológico, como proponen Choi y Pak (2008, E42), sus aportaciones ofrecieron al conocimiento de la antigüedad perspectivas que hubieran sido difíciles de alcanzar sin su formación.

Otros arqueólogos vocacionales más cercanos epistemológicamente hablando a la arqueología provinieron del derecho, la filología o la arquitectura. Un abogado cuyo conocimiento profesional estuvo en la base del estudio de las leyes municiples romanas encontradas en Málaga en 1851 sería Manuel Rodríguez de Berlanga (1825-1909) (ver artículo de M. ${ }^{a}$ José Berlanga en este dosier). Otros abogados que se interesaron por la arqueología fueron Manuel Oliver y Hurtado (18311892) y, más tarde, Ángel del Arco y Molinero (1862-1925) o Francesc Carreras i Candi (18621937). Filólogos hubo varios, sobre todo alemanes, y encontramos varios ejemplos en el artículo de Rafael Sabio González en este volumen. Son igualmente dos magníficos ejemplos Emil Hübner (1834-1901) o, de una época más tardía, tal como podemos leer en la aportación a este dosier de Ignasi Garcés y M. ${ }^{a}$ Paz Gómez, Adolf Schulten (1870-1960), sobre el que volveremos más adelante. Finalmente, desde el siglo XviıI, como demuesta Isabel Ordieres en este dosier, los arquitectos serían una pieza clave en la descripción de los monumentos antiguos. Esto es porque las ruinas y los edificios antiguos se percibieron, en su materialidad, como testigos del pasado nacional. Ordieres nos habla en su trabajo de arquitectos de la época ilustrada como el marqués de Valdeflores —Luis José de Velázquez de Velasco- o José Hermosilla, por citar a dos de ellos, y del xix como Manuel Assas, los hermanos de los Ríos o Ricardo Velázquez Bosco entre otros. 
Es interesante constatar, sin embargo, que no todas las colaboraciones posibles en aquel momento se produjeron en España, y esto se puede advertir bien realizando una comparación con Escandinavia. Allí, nos cuenta Kristian Kristiansen, se organizaron una serie de comisiones con un nombre muy poco elegante en su traducción al castellano: Comisión de los Montones creados por los Desechos de Cocina (køkkenmøddingkommission en danés, traducido al inglés como Kitchen Midden Commission). La primera de ellas, también llamada el Comité Lejre, tiene lugar entre 1848 y 1869, y se forma en el marco de la Academia Danesa de Ciencias y Letras como resultado del debate mantenido en 1848 en esta institución. Este surge a raíz de una presentación del naturalista Japetus Steenstrup relatando sus excavaciones de montículos formados por conchas de ostras. Formaron parte de la comisión el mismo Steenstrup junto con un geólogo, Forchammer, y un arqueólogo, Worsaae (Jens Jacob Asmussen Worsaae, 1821-1885). Este último, conservador del Museo Nacional de Dinamarca, era acérrimo defensor de la ciencia y del procedimiento científico frente a lo que él denominaba el mito histórico, y en esto coincidía con Steenstrup, pero ambos diferían en la lectura de la diferencia de la tipología del material arqueológico en cada estrato, que el naturalista explicaba por razones funcionales, mientras que Worsaae, quien finalmente tenía razón, las entendía como evolución tecnológica a lo largo del tiempo. Por otra parte, Steenstrup ofreció novedades importantes a la arqueología: en la zona pantanosa de Vidnesdam reconoció cinco estratos diferentes caracterizados por los restos de distintas especies de árboles: álamo, pino, roble (en este estrato también encontró un objeto arqueológico), aliso y haya, y sugirió que cada una de estas fases duraría unos dos mil años (Gron \& Rowley-Conwy 2018, 38). Los resultados de todos estos trabajos se conocieron casi inmediatamente en toda Europa gracias a las redes de contacto de entonces - correspondencia, conferencias de estos arqueólogos en otros países y publicaciones-. Así lo demuestran las referencias a su trabajo por parte de Mérimée y Lartet (Kristiansen 2002, 12-15). Pese a que algunos «arqueólogos» españoles se hallaban integrados en estas redes todavía quedan por identificar menciones a esta primera comisión. En Francia sí que las encontramos, pero lo cierto, sin embargo, es que tampoco parece que allí el conocimiento de la relación interdisciplinar inspirara otras del mismo corte, poniendo en otra luz la posible pasividad de España que entonces contaba con muchos menos medios que su vecino galo. Algo diferente pasaría en Suiza, donde sí que encontramos al menos a un botánico — Oswald Heer- y a un zoólogo — Ludwig Rütimeyer - trabajando en temas asociados con el medio ambiente prehistórico, en concreto sobre los restos que se encontraban en los yacimientos palafíticos (Delley \& Kaeser 2013). Este ejemplo nos indica que la interdisciplinariedad, aunque ayude, no se emula necesariamente por el conocimiento de su existencia. En este caso concreto quizá los especialistas en cada disciplina todavía eran demasiado poco numerosos en el caso de España como para permitir que se produjera un traspaso efectivo de la práctica interdisciplinaria a nuestro país.

En Dinamarca se establecería una segunda comisión hacia finales de siglo. En 1893 una crítica de un zoólogo a la división arqueológica de los periodos pre-metalúrgicos llevaría a otra comisión en la que como especialista botánico concurrirían Emil Rostrup (Kristiansen 2002, fig. 2.3) y Georg Sarauw (Kristiansen 2002, 19) en un contexto arqueológico ahora dominado por las figuras de Oscar Montelius en Suecia y Sophus Müller en Dinamarca impulsando ambos la sistematicidad en excavaciones y la imposición del método tipológico. El primero identificó los restos de plantas carbonizadas y Georg Sarauw reconoció una serie de plantas gracias a las impresiones observadas en cerámicas y por los granos encontrados (Gron \& Rowley-Conwy 2018, 39). El obligado abandono del Museo Nacional de este último investigador por el carácter inflexible de su director, Müller, impediría un desarrollo inmediatamente posterior de esta línea de trabajo (Kristiansen 2002, 19) y solamente en los años 1940 se retomaría parte de su investigación, ahora estudiando las plantas a través de análisis polínicos descubiertos treinta años antes (Kristiansen 2002, 20). En España pa- 
rece verse la influencia (¿directa o indirecta?) de estos trabajos en la relativa importancia que los hermanos Siret, como mencionaremos en el siguiente apartado, ofrecen a los restos biológicos.

\section{El Siglo xx ANTes de la Guerra Civil}

La primera parte del siglo xx en muchos aspectos representa una continuación del anterior. Seguimos encontrando una gran cantidad de naturalistas, geólogos o ingenieros como Lluís Marià Vidal i Carreras (1842-1922), Augusto de Gálvez-Cañero Alzoala (1878-1937), José Luis Martín Jiménez (1882-1928), Virgilio Viniegra de Vera (1889-1957), Manuel Cazurro (1894-1935) o Gabriel Puig y Larraz (1851-1917). También se interesan por la arqueología aquellos de antropología como Francisco de las Barras de Aragón (1869-1955), Bellas Artes y artistas como George Bonsor (1855-1930), Hermilio Alcalde del Río (1866-1947) o Manuel Gómez-Moreno (18701970), este último sirviendo como bisagra con la arqueología, como argumenta Francisco J. Moreno Martín en su trabajo para este dosier. Observamos también a los eclesiásticos con interés en historia del arte como forma de argumentar la naturaleza de la Biblia como fuente histórica y la autoridad de la propia Iglesia (ver Jordi Vidal en este volumen). También en estos ańos se desarrolla el turismo arqueológico, una actividad lúdica que también ayudan a desarrollar (Díaz-Andreu 2020, Gómez Díaz 2019).

No todos los que provienen de otras profesiones utilizan sus conocimientos para aportar visiones novedosas a la arqueología, como así lo vemos en el caso del médico José Galiay Sarañana (1880-1952), cuyos escritos están más relacionados con la historia del arte que con la antropología física. En este momento en el que las ciencias se están dividiendo existen personajes intermedios como Eduardo Hernández-Pacheco (1872-1965), el director de la Comisión de Investigaciones Paleontológicas y Prehistóricas desde su creación en 1914 y catedrático de la Facultad de Ciencias de Madrid, quien en los años diez y mitad de los veinte realizará una importante labor de investigación en prehistoria, que luego abandonará al chocar con Hugo Obermaier (Díaz-Andreu 2012b, 33-35). La creación para este último de una cátedra de prehistoria (oficialmente Historia Primitiva del Hombre) en 1922 y encuadrada una facultad de letras lleva consigo el traslado de esta subdisciplina, la arqueología prehistórica, de ciencias a humanidades, transformando de alguna manera a Eduardo Hernández-Pacheco de un profesional a un amateur, haciendo de alguna manera que sus aportaciones ya no se consideraran producidas desde dentro de la misma disciplina sino que fueran de carácter interdisciplinar. A esta situación él se negaría dando la espalda a los estudios prehistóricos, actitud que solo revertiría muy al final de su vida profesional.

En esta época de primeras décadas del siglo xx los mejores trabajos interdisciplinares dentro de la arqueología hispana los realizan extranjeros: Luis (Louis) Siret (1857-1934) y Adolf Schulten (1870-1960). El primero de ellos es un ingeniero de minas belga que acude por primera vez a España en 1881 con tan solo veinte años para reunirse con su hermano mayor Enrique (Henri), trabajando ambos para la Compañía de Minas de Sierra Almagrera en Almería y quedándose Luis ya a residir en este lugar durante toda su vida. Siret colaborará con otros especialistas. En su libro Les premiers ages du métal dans le Sud-Est de l'Espagne (Siret \& Siret 1887; 1890) dan importancia, quizá por influencia danesa como ya se ha adelantado, al descubrimiento de semillas carbonizadas. Estos materiales, depositados en Bruselas, serán estudiados con más detalle posteriormente por Friedrich (Fritz) Netolitzky (1935) y Maria Hopf (1990). Por otra parte, también colaboran con un médico, Victor D'Jacques, que les estudia los cráneos y, aunque de él poco sabemos, lo volvemos a encontrar mencionado por Cartailhac años después (Cartailhac 1892, 74-75). Como ha 
resaltado en un estudio reciente (Montero-Ruiz et al. 2011) Siret también analiza una serie de piezas del Bronce Final y realiza una serie de experimentos junto con Ramón de Cala y López, un farmacéutico que tenía un laboratorio minerológico en Cuevas, población cercana a donde vivía Siret mismo (Siret 1913).

Más sistemático que Siret en sus colaboraciones con miembros de otras disciplinas será el alemán Adolf Schulten quien, como se ha resaltado recientemente (Díaz-Andreu 2017), estudia en el momento en el que la arqueología interdisciplinar de campo se está desarrollando en las excavaciones de Olimpia entre 1875 y 1881. Garcés y Gómez Gonzalo detallan de forma excelente en su trabajo para este dosier las cooperaciones establecidas con profesionales de un amplio abanico de profesiones en tal cantidad de no tuvo precedentes en la arqueología española. Desgraciadamente tampoco tendría grandes seguidores, al menos de forma directa e inmediata, lo que se puede relacionar con su carácter huraño, por una parte, y por la otra por que sus coetáneos prestaron escasa atención a lo revolucionario de su actuación. De hecho, este particular no se resalta en los homenajes que le hacen en los años cuarenta, en los cuales, pese a que sí se citan colaboradores, no parece llamar la atención su existencia (Pericot 1940: 55, 59, 60, 61). García y Bellido, catedrático de Historia Antigua, lo percibe incluso como prueba de su incompetencia como excavador, explicando sus colaboraciones con estas palabras

Pero Schulten tuvo siempre conciencia exacta de estas lagunas en su formacion y no se arredró en casos importantes ante la necesidad de buscar el parecer de aquellos que pudieran opinar con mas autoridad que él. Schulten, pues, siguió siempre - al menos en los casos mas dificiles- el único proceder posible en un investigador consciente de su responsabilidad: la consulta de colegas especializados (García y Bellido 1960: 225).

\section{LA PRIMERA ÉPOCA FRANQUISTA (1939-1960)}

La fecha de 1960 de la última fuente citada y su total incomprensión hacia la interdisciplinariedad hace fácil entender que las conexiones entre personas de distintas disciplinas, por lo menos si estas se producían con cierta asiduidad, no fueran necesariamente bien vistas en la profesión. El bajo número de profesionales llevaba a que, sin embargo, todavía fueran importantes individuos que se ganaban la vida en otras profesiones y que no se habían especializado en la universidad en arqueología. Un ejemplo de ello es un médico entre las dos épocas Salvador Vilaseca i Anguera (1896-1975), o los ingenieros Jesús Cánovas Pesini (1900-1984) y Emeterio Cuadrado Díaz (1907-2002). Un caso especial puede ser el representado por Santiago Alcobé (1903-1977), catedrático de Antropología de la Universidad de Barcelona a quien «sus estudios de Paleoantropología le llevaron a una colaboración que puede calificarse de "cordial» con muy distinguidos arqueólogos y prehistoriadores y a participar en numerosos congresos de Prehistoria» (Font Serra 1979-80, 506). Escribiría libros como la Guía para el estudio antropológico de las poblaciones prehistóricas de España (Alcobé 1954) producido en el contexto del IV Congreso Internacional de Ciencias Prehistóricas y Protohistóricas celebrado en Madrid. Su alumno, Miquel Fusté, viajaría a París en 1951 para estudiar con el prof. Henri-Victor Vallois sobre poblaciones neolíticas en el Instituto de Paleontropología Humana (Calvo Calvo 1990, nota 72), lo que daría como resultado una extensísima cantidad de artículos que solo truncaría su temprano fallecimiento en 1967 (Ripoll Perelló 1966). En el otro extremo del espectro se encuentran los profesionales que en ocasiones solapan su quehacer con la arqueología, como los filólogos Antonio Tovar Llorente (1911-1985), Juan Álva- 
rez Delgado (1900-1987), José María Blázquez Martínez (1926-2016) o Darío Cabanelas Rodríguez (1916-1992).

Al mismo tiempo, sin embargo, sobre todo los prehistoriadores se van dando cuenta de que en otros países empieza a darse el caso de especialistas sin formación arqueológica universitaria expertos en alguna de lo que en un principio se llamaban ciencias auxiliares de la arqueología (véase Almagro en su célebre Introducción al estudio de la prehistoria ${ }^{2}$ (1960), que comienzan a integrarse profesionalmente en instituciones arqueológicas. Podemos citar tres casos de este tipo que los arqueólogos más importantes en España conocían bien: van Giffen, Zeuner, y Leroi-Gourhan. El holandés Albert Egges van Giffen (1884-1973), quien se había formado en zoología y biología, ocupa desde 1930 hasta su jubilación en 1954 cátedras de prehistoria en Groningen y Amsterdam ${ }^{3}$. A van Giffen le invita Julio Martínez Santa-Olalla a España en 1953 (Presedo 1955) y luego participa en la excavación de El Pendo (González Echegaray 1980, 21). Por su parte, Frederick Zeuner (1905-1963) es un geólogo aleman a quien el nacional socialismo le lleva a refugiarse en Inglaterra en 1934 y consigue ser profesor honorario de Geocronología en el Instituto de Arqueología de la Universidad de Londres, y finalmente obtener allí una cátedra en 1946 (Díaz-Andreu 2012a, 148-152; Simpson 2000-01). Como se percibe en la correspondencia con él mantenida, tanto Martín Almagro como Luis Pericot le trataron y por tanto fueron conscientes de su inserción profesional en una institución arqueológica, y la alta estima que tuvieron de su trabajo les llevó a ayudarle a publicar traducciones al castellano de sus dos libros más importantes (Zeuner 1956; 1959). El tercer investigador de formación en otra ciencia es André Leroi-Gourhan, aunque su caso es menos claro (ver también el trabajo de Palacio en este dosier). Es antropolólogo, pero trabaja a partir de 1946 en una institución con arqueólogos, el Musée de l'Homme. También imparte docencia en la Sorbona como catedrático de Etnología General y Prehistoria, donde se asume un puente interdisciplinar entre la etnología/antropología y la prehistoria. De estos tres ejemplos — van Giffen, Zeuner y Leroi-Gourhamconcluimos de forma semejante a la que lo hacíamos anteriormente en este artículo para otro caso: el conocimiento de casos en el extranjero en los que la interdisciplinariedad está llevando a nuevas relaciones entre las ciencias no significa que se produzca una imitación inmediata de las mismas. De nuevo la razón parece ser la misma: no existe suficiente peso numérico de profesionales en la arqueología española como para que haya posibilidad de integración de aquellos formados en otras ramas del saber en instituciones arqueológicas. Los pocos trabajos que iban apareciendo se guardaban para los pocos discípulos directos de los profesionales de entonces.

En todo caso, en este periodo del primer franquismo sí que aparece una cierta curiosidad por lo que las ciencias auxiliares pueden hacer por la arqueología que muestran los propios arqueólogos en sus escritos: así encontramos artículos sobre fotografía aérea (Almagro Basch 1943; Martínez Santa-Olalla 1942), climatología analizando moluscos (Pericot 1944), paleobotánica (Martínez Santa-Olalla 1946) y cualquier ciencia que esté relacionada con proveer de cronología absoluta a la prehistoria (Pericot 1949a; Pericot 1949b; Sáez Martín 1951). La correspondencia albergada en el Fons Pericot de la Biblioteca de Catalunya nos avisa de que los catedráticos en España estaban en contacto con colegas de otras ciencias como es el caso de Luis Pericot con el geólogo de la universi-

2 A partir de la segunda edición de 1963 el título de este libro pasa a ser Introducción al estudio de la prehistoria y de la arqueología de campo.

${ }_{3}^{3}$ Nos parece interesante reproducir aquí la percepción sobre este personaje que hace Waterbolk sobre van Giffen: siendo biólogo «van Giffen se especializó en geología costera y arqueozoología, pero siempre buscó la experiencia de paleobotánicos, científicos del suelo, antropólogos físicos y químicos. Su enfoque multidisciplinario de la arqueología ha servido ampliamente como modelo y es una segunda razón importante para su renombre internacional» (Waterbolk 1999, 336). 
dad de Columbia Helmut de Terra, o que las primeras fechas de carbono catorce se obtienen a finales de este periodo (Almagro Basch 1959).

Ya hemos mencionado que en el trabajo de campo existen equipos interdisciplinares en arqueología desde el siglo XIX, siendo adalid de esto los trabajos en Olimpia en la segunda mitad de los 1870. Falta conocer hasta qué punto esto tuvo continuidad tanto en Alemania como en otros países, pero en el caso de España vemos que será en estos momentos cuando se produzca el primer intento consciente de un arqueólogo español de realizar un equipo de campo interdisciplinar. Este será el intentando por Julio Martínez Santa-Olalla en El Pendo, a donde invita a excavar a un grupo de arqueólogos de otros países, cada uno con una especialidad, entre los que destacamos los arqueólogos André Cheynier y François Bordes para lítica, Arlette Leroi-Gourhan para palinología y Albert Egges van Giffen probablemente para arqueozoología. El fracaso esta indicado por el nulo rigor a la hora de publicar, puesto que de todos ellos solo sacaría a la luz algunos datos de lo realizado, y solo en forma de menciones, la arqueóloga francesa (González Echegaray 1980; 11).

\section{LA SEGUNDA ÉPOCA FRANQUISTA (1960-1975)}

Será a partir de los años sesenta cuando la interdisciplinariedad se produzca con más fuerza en España. Ahora es cuando los arqueólogos, primero tímidamente y luego con más asiduidad, comienzan a colaborar con especialistas en otras ramas del saber, y estos a su vez buscan a arqueólogos en busca de datos que ellos necesitan para sus investigaciones. En restos botánicos tenemos al ingeniero Ricardo Téllez (López García 1999) y en palinología a la geóloga Josefa Menéndez Amor (1916-1985), junto con su maestro, el holandés Frans Florschütz (1888-1965), cuyos trabajos comienzan a finales de los cincuenta (Perejón 1988). Los arqueólogos comienzan a colaborar con los físicos para la datación por radiocarbono ya desde finales de los años cincuenta (Almagro Basch 1959; Leisner \& Veiga Ferreira 1963; Schubart \& Pascual 1966). En Madrid el Laboratorio de Geocronología se funda en 1966 gracias a una beca de la Fundación Juan March (Rubinos Pérez 2002, 185). En él trabaja Fernán Alonso (1935-2002) quien producirá unas primeras fechaciones de radiocarbono en 1971 (Alonso et al. 1978), que en un primer momento producirán una cierta sorpresa. Como él mismo explicaba:

Cuando en 1971 obtuve varias fechas de carbono-14 en muestras de cereal de la Cueva de los Murciélagos (Córdoba), en asociación con cerámica a la almagra, las dataciones de alrededor del 4000 a.C. sorprendieron por ser casi 1000 años más antiguas de lo que parecía aceptar la cronología arqueológica del momento. Afortunadamente, otras muestras fueron enviadas (...) al laboratorio de Groningen, cuyas dataciones coincidieron con las anteriores, con lo cual la discusión sobre posibles errores experimentales, contaminaciones u otras pintorescas interpretaciones quedó zanjada (Alonso 1993; 3).

A pesar de los ejemplos citados, el interés de los arqueólogos españoles hacia la interdisciplinariedad distó mucho de lo que se estaba produciendo en otros países: según Alcina Franch, entre los años 1966 y 1971 solo se publicaron 18 trabajos científicos escritos por autores espańoles relacionados con ciencias como la geología, edafología, arqueozoología, arqueobotánica, radiocarbono, metalografía y antropología física en las cinco revistas más importantes del momento (Ampurias, Archivo de Prehistoria Levantina, Caesaraugusta, Pyrenae y Trabajos de Prehistoria) (Alcina Franch 2002). Un repaso a la serie Excavaciones Arqueológicas en España refuerza esto mismo, con conta- 
das excepciones, todas ellas en yacimientos de cronología paleolítica: la Cueva del Otero con un estudio paleontológico y otro palinológico (González Echegaray, García Guinea \& Begines Ramírez 1966), las clasificaciones de fauna realizadas por Altuna en las cuevas paleolíticas de Los Casares y de La Hoz (Beltrán Martínez \& Barandiarán 1964-65), la sedimentología, paleontología y antropología física de la Cueva de los Casares (en Riba de Saelices, Guadalajara) (Barandiaran Maestu 1973). La única excepción cronológica es un escueto estudio de huesos de animales en la publicación de Toscanos (Schubart, Niemeyer \& Pellicer 1969) y los análisis antropológicos de Tito Varela y al final del periodo (Varela 1975a; Varela 1975b).

En Alemania, en un desarrollo de una fase anterior menos conocida pero que debió de desarrollarse hacia los años veinte, siendo heredera de lo acontecido el siglo anterior, observamos un interés por parte algunos científicos hacia la arqueología. En lo que se refiere a España vemos que se producen a partir de 1960 una serie de estudios de metalurgia en la serie Metallanalysen Kupferzeitlicher und Frühbronzezeitlicher Bodenfunde aus Europas (Junghans, Sangmeister \& Schröder 1960; Junghans, Sangmeister, \& Schröder 1968-74). Por otra parte, aparece en 1969 el primer libro de la serie de Studien über frühe Tierknochenfunde von der Iberischen Halbinsel (es decir "Estudios sobre los hallazgos de fauna arqueológica en la Península Ibérica»), que publicaría cuatro volúmenes en esta época (Boessneck 1969; Driesch 1972; Schubart 1973; Uerpmann 1971) y ocho más en la siguiente, para dejar de publicarse en 1990. Además de los estudios de paleofauna, también se llevan a cabo estudios de paleobotánica por parte de Maria Hopf entre 1965 y el principio de los noventa (Peña-Chocarro \& Pérez-Jordà 2018, 9-10; Stika 2010). Ahora bien, el interés de algunos arqueólogos alemanes no significa que todos lo muestren, como se hace obvio al hojear publicaciones de excavaciones como el Cerro de la Virgen de Orce en Granada (Pellicer \& Schüle 1966) o S’Illot en Baleares (Rosselló-Bordoy \& Frey 1966). Desgraciadamente, tampoco es que el interés por lo interdisciplinario surtiera un efecto inmediato en los arqueólogos españoles, quizá porque los estudios se realizan sin la participación activa de jóvenes en formación y por lo tanto no se traspasa el conocimiento a las nuevas generaciones de profesionales en España.

Esta ausencia de traspaso de conocimiento también tendrá lugar hasta cierto punto en el caso de los equipos anglosajones que en esos años trabajan en España. El primero de ellos será el que excava en el yacimiento del paleolítico inferior soriano de Torralba y Ambrona en primer lugar entre 1961 y 1963, en 1973 y entre 1980 y 1983. El equipo dirigido por F. Clark Howell no solamente tiene a los citados Menéndez Amor y Florschütz, sino también al geólogo Karl Butzer y al paleontólogo de vertebrados Emiliano Aguirre, entre otros, pero su influencia en la arqueología profesional española será prácticamente nula, no porque no haya jóvenes en ellos, sino porque los pocos que colaboran con este equipo no logran luego profesionalizarse (Santonja 2019). Mayor suerte tiene el proyecto británico «The Early History of Agriculture» que discurre entre los años 1967 y 1976 y que incluye a España entre los países en los que actúa. Dirigido por Grahame Clark (19071995) y Eric Sidney Higgs (1908-1976), trae a la península a entonces estudiantes como Geoff Bailey e Iain Davidson, este último manteniendo con posterioridad una larga colaboración con arqueólogos valencianos que permitirá una transmisión de saberes que tendrá sus frutos en el siguiente periodo establecido en este artículo. El proyecto era una aplicación de las teorías de la arqueología espacial y, aunque al parecer no de forma consciente (Burillo Mozota 2005), influye en el entonces joven Francisco Burillo, que hacía las veces de inspector por parte española (Díaz-Andreu 2012a; 212), y quien, ya como docente universitario, desarrollará una serie de actividades relacionadas con la arqueología espacial en el siguiente periodo.

En el mundo anglosajón el interés por la interdisciplinariedad está conectado con un movimiento teórico surgido en Estados Unidos que terminará cuajando en los años sesenta bajo la de- 
nominación de Nueva Arqueología. Los actores de nuestros dos proyectos del párrafo anterior no provienen del núcleo duro del movimiento formado por Lewis Binford y sus antiguos alumnos de la Universidad de Chicago de principios de los sesenta, pero responden a la misma filosofía: dar prioridad a la teoría, el método y la técnica a la hora de desarrollar proyectos, prestar cierta atención pero sin enfocarse en tipologías ni obsesionarse con cronología, y realizar interpretaciones funcionales que tengan en cuenta el contexto geográfico, social, ecológico y económico y la función de objetos, sitios y paisajes arqueológicos (Díaz-Andreu en prensa). Una versión suave de la Nueva Arqueología influiría en España solo tras el cambio político ocurrido tras la muerte del dictador Francisco Franco en 1975, algo que relatamos en el siguiente apartado.

\section{LA NORMALIZACIÓN DE LA INTERDISCIPLINARIEDAD}

Víctor Fernández apuntaba hace unos pocos años que la Nueva Arqueología tenía tres niveles que iban de más metodológico a más teórico, lo que se correspondía a una aceptación de más a menos en la disciplina. En el nivel más bajo y con mayor aprobación en la disciplina, se encuentra la integración de métodos y prácticas provenientes de las ciencias naturales con sus regularidades implícitas, cuantificación y modelado de datos. En un segundo nivel el autor coloca el retorno al evolucionismo que lleva a una nueva recepción de los principios universales en el comportamiento humano y una vuelta a la etnoarqueología y en el tercero coloca la recepción de la teoría en el discurso arqueológico (Fernández Martínez 2016, 100-101). Es el primer nivel, el metodológico, el mejor recibido no solo en Espańa sino en general en todo el mundo occidental, y ello tendría consecuencias a la postre para un apoyo sin precedentes a la interdisciplinariedad en arqueología.

La Nueva Arqueología se introduce en España por varios caminos. En primer lugar empiezan a llegar los escritos producidos en Estados Unidos en traducciones que publican los miembros del Departamento de Antropología Americana de la Universidad Complutense de Madrid en la revista Cuadernos de Antropología Social y Etnología entre 1972 y 1974 y como libros (Clarke 1977; Chang 1976; Hole \& Heizer 1977 (1965); Watson, Leblanc \& Redman 1974) —aunque, curiosamente, a Binford no se le tradujo hasta más tarde (Binford 1988)_. Esta influencia se ve reflejada en la revista Trabajos de Prehistoria, en la que desde 1982 cada número incluye por lo menos un artículo con referencias a autores encuadrados en la Nueva Arqueología (Fernández Martínez 2016, 105). Por otra parte, ya hemos adelantado en el apartado anterior la influencia que pudo tener en Burillo su conexión con el proyecto «The Early History of Agriculture» de Cambridge y que daría sus frutos en 1984 con el primero de los Congresos de Arqueología Espacial en Teruel, que se seguirían celebrando hasta 2010 dando lugar a un total de 28 volúmenes de trabajos.

Es necesario apuntar, sin embargo, que cierta precaución se hace necesaria en relación al papel de la Nueva Arqueología para la transformación de la arqueología en esos años, y para la normalización de la interdisciplinariedad, puesto que muchos de los protagonistas de este cambio citan además otras fuentes. Entre los mismos alemanes en España, más impermeables en estos años a la influencia anglosajona, se normaliza la interdisciplinariedad, siendo las excavaciones de Fuente Álamo un ejemplo de ello. Como relataba Mauro Hernández sobre Hermanfrid Schubart:

En las excavaciones [de Fuente Álamo]... fueron concebidas como una colaboración entre colegas de distintas nacionalidades y campos de investigación como la antropología física, edafología, 
antracología, carpología, cambio de las líneas costeras, criaderos de minerales, análisis de metales, petrología, arqueozoología y dataciones absolutas. Como había ocurrido ańos antes en Zambujal, el yacimiento almeriense se convertiría en una excepcional escuela de la arqueología de campo (Hernández Pérez 2006, 56).

Un momento clave en el proceso de la normalización de la interdisciplinariedad fueron las primeras jornadas de metodología de la investigación prehistórica de Soria 1981. Como estudiante de carrera en aquellos ańos, una de las autoras de este artículo (Díaz-Andreu) se acuerda de los comentarios realizados por profesores que no se identificaban en su gran mayoría con la Nueva Arqueología sobre las jornadas y de la sensación que nos transmitían aquellos que allí habían estado de haber participado en un evento de principal importancia para la arqueología en España. Las comunicaciones de Soria habían tratado de terminología, epistemología, geomorfología, cronología, biometría y tipología incluyendo materiales menos tratados como el hueso y proponiendo ángulos novedosos a aspectos más tradicionales. Además, otros se centraron en geofísica, edafología, paleontología, palinología, arqueometalurgia, análisis económico, demografía, arqueología espacial, estratigrafía y fotografía (Jornadas..., 1984).

Uno de los participantes en aquellas jornadas, Salvador Rovira ${ }^{4}$, explicaba así años después por qué se había especializado en arqueometalurgia en los años ochenta:

El que me convirtiera en un arqueometalúrgico fue otra jugarreta del destino. En 1982 el entonces joven Subdirector General de Arqueología..., Manuel Fernández-Miranda, junto con Germán Delibes, Concepción Martín, M. ${ }^{a}$ Dolores Fernández-Posse (Pachula) y M. ${ }^{a}$ Luisa Ruiz-Gálvez estaban dando forma a un Proyecto de Arqueometalurgia de la Península Ibérica. En España no había tradición en investigación arqueometalúrgica. Salvo algunos trabajos puntuales y aislados sobre estudios de metales antiguos, la mayoría de ellos desarrollados en el CENIM-CSIC, y algunas meritorias aportaciones de los laboratorios del entonces Instituto de Conservación y Restauración de Obras de Arte (ICROA), el panorama resultaba un desierto.

La situación en el resto de Europa era bien distinta, pues, desde los años 1950, el surgimiento de la Arqueometria, al socaire de las propuestas de una Nueva Arqueología y de una Arqueología Analítica, ya estaban dando sus frutos que cristalizaban en revistas especializadas como Archaeometry, Revue d'Archéométrie, Der Anschnitt, Archaeomaterials, por mencionar sólo unas pocas. Asimismo, periódicamente se organizaban congresos internacionales en los que la Arqueometalurgia era parte importante cuando no especifica. Tanto en unas como en los otros la participación española prácticamente brillaba por su ausencia.

Entre los objetivos del mencionado Proyecto estaba el ir cambiando esa situación. Fue entonces, en 1982, cuando se me invitó a integrarme en el equipo humano para hacerme cargo de una inicial célula de análisis de materiales a la que José M. ${ }^{a}$ Cabrera, entonces director del ICROA, dio cobijo en el laboratorio de dicho Instituto (Rovira 2010-11, 45-46).

«Ir cambiando esa situación» era algo que a nivel político estaba ocurriendo en España en aquellos años, donde muchas cosas estancadas durante décadas estaban transformándose. Esto también se reflejó en jóvenes entonces recién graduados, como fue el caso en Cataluña del Grup Centvint.

\footnotetext{
4 Salvador Rovira fue el que introdujo a una de las autoras de este artículo, Margarita Díaz-Andreu, a las ideas de la Nueva Arqueología, prestándole multitud de libros publicados por algunos de los principales adalides
}

de la formación. Esto resalta aún más el hecho de que el papel de esta corriente teórica quede hasta cierto punto minimizado al explicar lo que le llevó a trabajar en arqueometalurgia. 
En 1983 este grupo, que había tomado este nombre de una cueva denominada con este numeral (Cova 120), explicaba que:

Actualmente, en arqueología se están generalizando los trabajos multidisciplinarios o interdisciplinarios, y se toma esta relación de disciplinas como principal innovación metodológica, y como razonamiento de la existencia del equipo. Es evidente que durante todo el proceso no todos hacemos el mismo trabajo; dentro de este mismo nivel de división de trabajos situamos, el Cent-Vint Group [sic], la división interdisciplinaria. Cada uno de los miembros, además de ser arqueólogo, es especialista en un aspecto diferente (palinología, antracología, antropología física...) y realiza los análisis concretos. Esta separación existe porque, así como los demás trabajos pueden realizarlos espontáneamente un miembro u otro, en estas técnicas, por su compleja metodología, es más práctico que cada miembro trabaje siempre en la misma; esto no implica ninguna división en el trabajo (Grup Centvint 1983, 41, traducción nuestra).

El grupo había nacido en 1981, en las turbulencias e ilusiones de la transición política, algo que se refleja en la última frase del extracto citado y en su autodefinición en el artículo como grupo autogestionado. En 1984 publicaron un monográfico en la revista Cota Zero en la que se incluyeron artículos sobre palinologia (Jossette Renault-Miskovsky, Francesc Burjachs i Casas), macrorrestos (Willem van Zeist, Ramon Buxó), antracología (Jean-Louis Vernet, M. Teresa Ros), micromamíferos (Jean Chaline, Gabriel Alcalde), peces (Jean Desse, Georges Desse, Núria Juan-Muns i Plans) y macromamíferos (Jordi Estévez Escalera, John Watson), paleoecología (Paul G. Bahn y varios) y terminaban con un artículo sobre la arqueología teórica francesa frente a los métodos anglosajones escrito por Alain Gallay (Dossier-Mètodes científics... 1984).

La novedad en este periodo la constituye que estos jóvenes no solo se forman en su periodo doctoral en otras técnicas, sino que también logran integrarse en departamentos de arqueología. Es decir, lo que se produce no es estrictamente hablando interdisciplinariedad sino pluridisciplinariedad, puesto que integran varias disciplinas en una misma para albergarla en los departamentos de arqueología. No es un paso tan radical como lo que había pasado en Holanda, Inglaterra y Francia a partir de la II Guerra Mundial con especialistas en otros saberes formando parte de instituciones de arqueología, pero es un paso intermedio nunca experimentado antes en España. Haría falta un estudio exhaustivo para poder desentrañar todos los hilos de una gran madeja de diferentes especialidades, pero creemos no confundirnos al identificar entre los primeros pasos hacia esta nueva situación la creación en 1977 de un laboratorio de palinología integrado en el Instituto de Prehistoria del CSIC de Madrid. A este ejemplo aislado, personalizado en la figura de Pilar López García, se unirán muchos otros a partir de los ochenta y sobre todo en los noventa y se generaliza en los inicios del siglo XXI: arqueólogos graduados en la disciplina se especializan, en muchas ocasiones en el extranjero, en disciplinas tan diversas como la metalurgia, zoología, botánica, geología, antropología física, paleopatología, química, etc., para entonces incorporarse de nuevo en sus departamentos de origen, ahora como profesionales. A finales de los 1980 podíamos leer en un trabajo sobre la arqueología ante las ciencias auxiliares:

está claro que la investigación arqueológica si quiere sobrevivir en el siglo XxI debe utilizar los avances técnicos que nuestro siglo le ofrece cada vez con mayor rapidez. Como consecuencia se añade un problema adicional: debemos conocer qué técnicas nos van a ser útiles, en qué se basan, cuál es la idónea en cada momento, qué límites tiene... ¿Significa eso que debemos tener un montón de técnicos de otros campos a nuestro alrededor (suponiendo que los haya), acumular los trabajos individuales de estos técnicos y ser nosotros los compiladores? (Vila \& Estévez 1989, 272). 
Defendían estos autores que un

camino más laborioso, difícil, pero finalmente más rentable para conseguir una disciplina integrada y crítica, es que los propios laboratorios de arqueología sean los que realicen estros trabajos técnicos. Deben ser los propios arqueólogos quienes se adapten a las necesidades de su disciplina (Vila \& Estévez 1989, 273).

Y apoyaban la constitución de «laboratorios de Arqueología» con arqueólogos-técnicos en las distintas especialidades y adelantaban que se hacía necesario un replanteamiento en la formación de los estudiantes universitarios. En el siglo Xxi los deseos así expresados ya se han cumplido y la nueva composición de muchos de los departamentos universitarios así lo demuestran — Barcelona, Granada o Valencia, entre otros, son buenos ejemplos de esta nueva forma de entender la disciplina- En todo caso esto no impide que continúe la colaboración con otros colegas de otras especialidades, como así nos demuestran, serán conferencias interdisciplinares como la dedicada a debatir el paisaje mediterráneo a través del espacio y del tiempo en la que coincidieron arqueólogos, climatólogos, geógrafos, ingenieros, etc. (Ibáñez, Valero Garcés \& Machado 1997).

\section{Conclusiones}

Este artículo, tras repasar lo que se entiende por interdisciplinariedad y por otros términos afines, ha realizado un repaso diacrónico sobre cómo se ha producido esta en la arqueología en España. Hemos establecido varias épocas, empezando por el siglo xIX y distinguiendo en el xx cuatro etapas, la última de ellas extendiéndose hasta la actualidad. En nuestro relato hemos hecho especial énfasis en la interdisciplinariedad entre la arqueología y las ciencias epistemológicamente más apartadas - las ciencias naturales y exactas-, aunque hayamos mencionado otras más cercanas situadas en las humanidades. Nuestra narración ha incidido en el retraso inicial en las relaciones interdisciplinares en un sentido estricto en relación a otros países, aunque sensu lato estuvieron allí desde el principio. Con ello queremos apuntar que desde los inicios de los estudios arqueológicos aparecen individuos con conocimientos en otras ramas de la ciencia que pudieron ofrecer interpretaciones del pasado novedosas precisamente gracias a su formación y práctica profesional no relacionada con las antigüedades, pero sin que, sin embargo, esto llevara al traspaso de conocimientos entre unos y otros especialistas. En la gran mayoría de los casos esta ausencia de transferencia se debió fundamentalmente a la poca profesionalización del saber arqueológico, tanto porque el enfoque de los profesionales fuera limitado (en gran parte del XIX estos solo prestaron atención a lo romano e ignoraron los restos materiales sobre todo de época prehistórica), como porque el número de arqueólogos fuera demasiado bajo. Estos factores irán desapareciendo a lo largo del siglo xx siendo solo en el último cuarto de esta centuria cuando vemos normalizarse la interdisciplinariedad, o más bien la pluridisciplinariedad, en arqueología. En realidad, lo hace de una manera muy concreta: en un contexto político en el que todo cambio con respecto a lo anterior se valora como positivo, aunque continúe una cierta colaboración con miembros de otras ciencias, lo que se prima es especializar a nivel postdoctoral a los futuros profesionales arqueólogos en las otras ramas del saber para entonces integrarles profesionalmente en los propios departamentos de arqueología.

Hoy en día encontramos arqueólogos y arqueólogas que se dedican a un sinfín de especialidades: arqueobotánica en sus diferentes versiones — carpología o la palinología, etc.—, arqueometalurgia, arqueopetrología, arqueozoología, geoarqueología, paleopatología, o tecnología entre los 
más científicos y filólogos, estudiantes de literatura e historiadores de arte entre los más humanísticos, lista no exhautiva de todas las combinaciones posibles. El enfoque tomado en este artículo introductorio al dosier nos ha permitido dilucidar cómo se ha llegado a esta situación que, como las anteriores, es histórica y variará sin duda alguna en las décadas por venir. Ya lo hemos comentado al principio de esta introducción: la definición de la misma disciplina es contingente, ya que depende de la época, de los intereses de los científicos, de las tradiciones disciplinares y nacionales. Lo que vaya a pasar con la interdisciplinariedad solo el tiempo lo dirá.

\section{BibLiOgRAFÍA}

Alcina Franch, J., 2002, "José Alcina Franch: curriculum vitae (1922-2001)», Revista Española de Antropología Americana 68 (32), 13-68.

Alcobé, S., 1954, Guía para el estudio antropológico de las poblaciones prehistóricas de España, Congreso Internacional de Ciencias Prehistóricas y Protohistóricas, Madrid.

Almagro Basch, M., 1943, «La colaboración de la aviación militar en el campo de la Arqueología», Ampurias $5,247-251$.

Almagro Basch, M., 1959, «La primera fecha absoluta para la cultura de Los Millares a base de Carbono 14», Ampurias 21, 249-251.

Almagro Basch, M., 1960, Introducción al estudio de la Prehistoria, Madrid: Guadarrama.

Alonso, F., 1993, "Carbono 14: calibrar o no calibrar», Arqcrítica 5, 3-4.

Alonso, F., V. Cabrera, T. Chapa \& M. Fernández-Miranda, 1978, «Índice de fechas arqueológicas de C 14 en España y Portugal», en M. Almagro Gorbea \& M. Fernández-Miranda (eds.), C 14 y prehistoria de la Peninsula Ibérica, Madrid: Fundación Juan March (Serie Universitaria, 77), 155-183.

AlvargonzÁlez, D., 2011, «Multidisciplinarity, Interdisciplinarity, Transdisciplinarity, and the Sciences», International Studies in the Philosophy of Science 24 (4), 387-403.

Ayarzaguena SAnz, M., 2018, «El papel desempeñado por geólogos e ingenieros de minas en la consolidación de la Arqueología prehistórica en España (1860-1970)», Boletín Geológico y Minero 1129 (1-2), 13-33.

Barandiaran Maestu, I., 1973, La Cueva de los Casares (en Riba de Saelices, Guadalajara). Excavaciones Arqueológicas en España 76, Madrid: Ministerio de Educación y Ciencia. Dirección General de Bellas Artes. Servicio Nacional de Excavaciones Arqueológicas.

Beltrán Martínez, A., \& I. Barandiarán, 1964-65, Avance al estudio de las cuevas paleolíticas de Los Casares y de La Hoz. Excavaciones Arqueológicas en España 64, Madrid: Ministerio de Educación Nacional. Dirección General de Bellas Artes. Servicio Nacional de Excavaciones Arqueológicas.

Binford, L. R., 1988, En busca del pasado [Traducción de (1983). In Pursuit of the Past], Barcelona: Crítica.

Boessneck, J., 1969, Die Knochenfunde vom Cerro del Real bei Galera (Prov. Granada). Studien über frühe Tierknochenfunde von der Iberischen Halbinsel 1, München, Madrid: Institut für Palaeoanatomie, Domestikationsforschung und Geschichte der Tiermedizin der Universität München; DAI-Abteilung Madrid, 1986.

Burillo Mozota, F., 2005, «Prólogo», en: L. García Sanjuan (ed.), Introducción al reconocimiento y análisis arqueológico territorio, Barcelona: Ariel.

Calvo Calvo, L., 1990, «La antropología biológica en Cataluña», Llull 13, 321-348.

Callmer, J., M. Meyer, R. Struwe \& C. Theune-Vogt (eds.), 2006, The beginnings of academic pre-and protohistoric archaeology (1830-1930) in a European perspective, Berlin: Verlag Marie Leidorf (Berliner Archäologische Forschungen 2).

Cartailhac, É., 1892, Monuments primitifs des Îles Baleares, Toulouse: Privat.

Clarke, D. L., 1977, Arqueología Analítica [traducción de Clarke, D. L. (1968). Analytical Archaeology. London, Methuen], Barcelona: Editorial Belllaterra. 
Chang, K. C., 1976, Nuevas perspectivas en Arqueología [Chang, K. C. (1967). Rethinking archaeology New York, Random House], Madrid: Alianza Editorial.

Chor, B. C. K., \& A. W. P. РАK, 2006, «Multidisciplinarity, interdisciplinarity and transdisciplinarity in health research, services, education and policy: 1 . Definitions, objectives, and evidence of effectiveness", Clinical and Investigative Medicine 29, 351-364.

Chог, B. C. K., \& A. W. P. РАк, 2008, «Multidisciplinarity, interdisciplinarity, and transdisciplinarity in health research, services, education and policy: 3. Discipline, inter-discipline distance, and selection of discipline», Clinical and Investigative Medicine 31 (1), E41-8.

Delley, G., \& M.-A. Kaeser, 2013, «Archéologie et botanique: un aller-retour Suisse-Egypte en classe diachronique», en: C. Jacquat \& I. Rogger (eds.), Fleurs des pharaons, Hauterive: Laténium, 113-131.

DíAZ-Andreu, M., 1995, "Arte y arqueología: la larga historia de una separación", en: VII Jornadas de Arte. Historiografía del Arte español en los siglos XIX y XX, Madrid: Alpuerto, 151-160.

Díaz-Andreu, M., 2007, A World History of Nineteenth-Century Archaeology. Nationalism, Colonialism and the Past. Oxford Studies in the History of Archaeology, Oxford: Oxford University Press.

Díaz-Andreu, M., 2012a, Archaeological encounters. Building networks of Spanish and British archaeologists in the 20th century, Newcastle: Cambridge Scholars.

Díaz-Andreu, M., 2012b, "A Hundred years of post-Palaeolithic rock art studies in Spain / Cien años en los estudios de pintura rupestre post-paleolítica en la investigación española», en: J. J. García Arranz, H. Collado, \& G. Nash (eds.), The Levantine Question: the development of Spanish Levantine Rock-art, Budapest: Archaeolingua, 23-53.

DíAz-Andreu, M., 2017, "Contextualizando a Adolf Schulten», en: E. Baquedano \& M. Arlegui (eds.), Schulten y el descubrimiento de NVMANTIA, Alcalá de Henares: Museo Arqueológico Regional de la Comunidad de Madrid, 30-45.

Díaz-Andreu, M., 2020, A History of Archaeological Tourism. Pursuing leisure and knowledge from the eighteenth century to World War II. New York: Springer.

Díaz-Andreu, M. (en prensa), «Towards Archaeological Theory: a history», en: P. Díaz-del-Río, K. Lillios, \& I. Sastre (eds.), The Power of Reason, the Matter of Prehistory. Papers in Honour of Antonio Gilman Guillén, Madrid: Consejo Superior de Investigaciones Científicas (Bibliotheca Praehistorica Hispana).

Díaz-Andreu, M., G. Mora \& J. Cortadella (eds.), 2009, Diccionario Histórico de la Arqueología en España (siglos $X V-X X$ ), Madrid: Marcial Pons.

Dossier-Mètodes científics... 1984, «Dossier-Mètodes científics aplicats a la reconstrucció paleoambiental de la prehistòria", Cota Zero 4, 1-106.

Driesch, V. D., 1972, Osteoarchäologische Untersuchungen auf der Iberischen Halbinsel. Studien über frühe Tierknochenfunde von der Iberischen Halbinsel 3, München: Institut für Palaeonatomie, Domestikationsforschung und Geschichte der Tiermedezin der Universität München.

Fernández Martínez, V., 2016, "Learning to be scientific. The introduction of "New Archaeology" in Spain, 1975-1990", en: G. Delley, M. Díaz-Andreu, F. Djindjian, V. Fernández, A. Guidi \& M. A. Kaeser (eds.), History of Archaeology - international perspectives, Oxford: Archaeopress (British Archaeological Reports), 99-110.

Font Serra, A., 1979-80, «Prof. Santiago Alcobé Noguer (1903-1977)», Ampurias 41-42, 505-506.

García y Bellido, A., 1960, «Adolf Schulten», Archivo Español de Arqueología 33 (101-102), 222-228.

Goberna, M. V., 1986, «Los estudios de Prehistoria durante la segunda mitad del siglo xix y primeros años del xx - La obra de Luis Siret», en: Homenaje a Luis Siret, Sevilla: Junta de Andalucía, 28-34.

Gómez DíAz, A., 2019, "Arqueología y turismo arqueológico en la Exposición Iberoamericana de Sevilla. Jorge Bonsor y el Castillo de Mairena del Alcor», en: A. Graciani García (ed.), El turismo y la Exposición Iberoamericana de Sevilla. Vol. I. Oportunidades, promoción, imagen e identidad. Sevilla: Editorial Universitaria de Sevilla: 129-150.

González Echegaray, J., 1980, El yacimiento de la cueva de "El Pendo»: (excavaciones 1953-57). Bibliotheca Praehistorica Hispana 17, Madrid: Consejo Superior de Investigaciones Científicas, Instituto Español de Prehistoria; Departamento de Prehistoria de la Universidad Complutense. 
González Echegaray, J., M. Á. García Guinea \& A. Begines Ramírez (eds.), 1966, Cueva del Otero [Con la colaboración de Benito Madariaga de la Campa y Arlette Leroi-Gourhan], Madrid: Ministerio de Educación Nacional. Dirección General de Bellas Artes. Servicio Nacional de Excavaciones Arqueológicas (Excavaciones Arqueológicas en España 53).

Gracia Alonso, F., \& J. M. Fullola Pericot, 2005, «El Graduado Superior en Arqueología. Balance de una experiencia docente en la Universidad de Barcelona (2000-2005)», Complutum 16, 245-254.

Gron, K. J., \& P. Rowley-Conwy, 2018, "Environmental Archaeology in Southern Scandinavia», en: E. Pişkin, A. Marciniak, \& M. Bartkowiak (eds.), Environmental Archaeology. Current Theoretical and Methodological Approaches, New York: Springer (Interdisciplinary Contributions to Archaeology), 35-74.

Grup Centvint, 1983, «El treball arqueològic del cent-vint grup en objectius i plantejaments», Gra de Fajol 7, 41-42.

Halbertsma, R. B., 2008, «From Distant Shores: Nineteenth-Century Dutch Archaeology in European Perspective», en: N. Schlanger \& J. Nordbladh (eds.), Histories of Archaeology: archives, ancestors. practices, Oxford: Berghahn Books, 21-35.

Hernández Pérez, M. S., 2006, "Hermanfrid Schubart y la Prehistoria de la Península Ibérica», en: M. H. Olcina Doménech \& J. A. Soler Díaz, Arqueología en Alicante. Homenaje a Hermanfrid Schubart, Alicante: Museo Arqueológico de Alicante-MARQ, 50-57.

Hole, F., \& R. F. Heizer, 1977 (1965), Introducción a la Arqueología Prehistórica, México City: Fondo de Cultura Económica.

Hopf, M., 1990, «Kulturpflanzenreste aus der Sammlung Siret in Brüssel», en: H. Schubart \& H. Ulreich (eds.), Die Funde der sudöstspanischen Bronzezeit aus der Sammlung Siret, Mainz am Rhein: Verlag Phillipp von Zabern (Madrider Beiträge 17), 397-413.

Ibáñez, J. J., B. L. Valero Garcés \& C. Machado (eds.), 1997, El paisaje mediterráneo a través del espacio $y$ del tiempo, Logroño: Geoforma Ediciones.

Jensen, O. W., 2004, «Earthy Practice. Towards a History of Excavation in Sweden, in the 17th and 18th centuries», Current Swedish Archaeology 12, 61-82.

Jornadas, 1984, Primeras jornadas de metodología de investigación prehistórica. Soria 1981, Madrid: Ministerio de Cultura, Instituto de Conservación y Restauración de Bienes Culturales.

Junghans, S., E. SAngmeister \& M. SChröDer, 1960, Metallanalysen Kupferzeitlicher und Frühbronzezeitlicher Bodenfunde aus Europas. Studien zu den Anfängen der Metallurgie 1, Berlin: Gebr. Mann.

Junghans, S., E. Sangmeister \& M. Schröder, 1968-74, Kupfer und Bronze in der Fruhen Metallzeit Europas. 4 vols. Studien zu den Anfängen der Metallurgie 2, 3. Berlin: Gebr. Mann.

Klein, J. T., \& W. Newell, 1997, «Advancing Interdisciplinary Studies», en: J. G. Gaff and J. L. Ratcliff (eds.), Handbook of the Undergraduate Curriculum: A Comprehensive Guide to Purposes. Structures, Practices, and Change, San Francisco: Jossey-Bass, 393-415.

Klindt-Jensen, O., 1975, A History of Scandinavian Archaeology, London: Thames and Hudson.

Kristiansen, K., 2002, "The Birth of Ecological Archaeology in Denmark: History and research environments 1850-2002», en: A. Fischer and K. Kristiansen (eds.), The Neolithisation of Denmark. 150 years of debate, Poole: Orca Book, 11-31.

Leisner, V., \& O. d. Veiga Ferreira, 1963, «Primeiras datas de radiocarbono 14 para a cultura megalitica portuguesa", Revista de Guimaraes 73, 358-366.

López García, P., 1999, «Ricardo Téllez (1913-1998)», Trabajos de Prehistoria 56, 7-8.

Martínez Santa-Olalla, J., 1942, "La aviación al servicio de la arqueología», Atlantis. Actas de la Sociedad de Antropología, Etnografía y Prehistoria 18, 127-164.

Martínez Santa-Olalla, J., 1946, "Cereales y plantas de la cultura ibero-sahariana», Cuadernos de Historia Primitiva 1, 35-45.

Montero-Ruiz, I., C. Cacho, E. Galán, O. García-Vuelta \& M. Murillo-Barroso, 2011, «Luis Siret: arqueólogo experimental», en: M. Sánchez Romero (ed.), Memorial Luis Siret. I Congreso de Prehistoria de Andalucía, Sevilla: Junta de Andalucía, 529-532. 
Netolitzky, F., 1935, «Kulturpflanzen und Holzreste aus dem Prähistorischen Spanien und Portugal», Buletinul Facultății de Ştiinţe din Cernăuţi [Boletín de la Facultad de Ciencias de Chernivtsi, Rumanía] IX, 4-8.

Pellicer, M., \& W. Schüle, 1966, El Cerro de la Virgen de Orce (Granada). Excavaciones Arqueológicas en España 46, Madrid: Ministerio de Educación Nacional. Dirección General de Bellas Artes. Servicio Nacional de Excavaciones Arqueológicas.

Peńa-Chocarro, L., \& G. Pérez-Jordà, 2018, «Los estudios carpológicos en la Península Ibérica: un estado de la cuestión", Pyrenae 49 (1), 7-45.

Perejón, A., 1988, "Josefa Menéndez Amor (1916-1985)», Boletín Real Sociedad Española de Historia Natural 84, 53-60.

Pericot, L., 1940, «Adolfo Schulten - su vida y sus obras», en: L. Pericot (ed.), Homenaje de la Universidad de Barcelona a su Doctor Honoris Causa con motivo de su 71 aniversario, Barcelona: Universidad de Barcelona (Anales de la Universidad de Barcelona. Memorias y Comunicaciones), 45-76.

Pericot, L., 1944, «Un nuevo procedimiento para el estudio climático por el análisis de los suelos», Ampurias VI, 285.

Pericot, L., 1949a, «La datación de los huesos prehistóricos por su contenido en fluorina», Ampurias XI, 176.

Pericot, L., 1949b, «Una rectificación a la larga cronología del Magdaleniense». Ampurias XI, 178-179.

Presedo, F., 1955, «Primer curso internacional de Arqueología de Campo», Noticiario Arqueológico Hispánico II, 252-255.

Puche Riart, O., 2002, «La contribución de los ingenieros a la arqueología española», en: S. Quero Castro, A. Pérez Navarro (eds.), Historiografía de la Arqueología Española. Las Instituciones, Madrid: Museo de San Isidro, 13-46.

Querol, M. A., 2005, «La génesis del título universitario de arqueología: Desde mi ángulo», Complutum $16,213-219$.

Ripoll Perelló, E., 1966, «Necrología. Prof. Miguel Fusté Ara (1919-1966)», Ampurias XXVIII, 309-311.

Rosselló-Bordoy, G., \& O. H. Frey, 1966, Levantamiento planimétrico de «S'Illot» (San Lorenzo-Mallorca). Excavaciones Arqueológicas en España 48, Madrid: Ministerio de Educación Nacional. Dirección General de Bellas Artes. Servicio Nacional de Excavaciones Arqueológicas.

Rovira, S., 2010-11, «La Arqueometalurgia y sus problemas: Algunas reflexiones personales», Boletín de la Sociedad Española de Amigos de la Arqueología 46, 275-285.

Rubinos Pérez, A., 2002, «D. Fernán Alonso Matthias (1935-2002)», Archivo Español de Arqueología 75, 185-186.

Sáez Martín, B., 1951, "La cronología arqueológica por medio del carbono radioactivo», Atlantis. Actas y Memorias de la Sociedad Española de Antropología, Etnografía y Prehistoria XXVI, 59-66.

Santonja, M., 2019, «El desarrrollo de la investigación en los yacimientos Paleolíticos de Torralba y Ambrona (Soria, España) a partir de los diarios inéditos de Dolores Echaide», Munibe 70, 5-19.

Schubart, H., 1973, Tierknochen von westphönizischen und phönizisch beeinflußten Ansiedlungen im südspanischen Küstengebiet. Studien über frühe Tierknochenfunde von der Iberischen Halbinsel 4, München, Madrid: Institut für Palaeoanatomie, Domestikationsforschung und Geschichte der Tiermedizin der Universität München; DAI-Abteilung Madrid.

Schubart, H., H. G. Niemeyer \& M. Pellicer, 1969, Toscanos. La factoría paleopúnica en la desembocadura del río de Vélez. Excavaciones de 1964. Excavaciones Arqueológicas en España 66, Madrid: Ministerio de Educación y Ciencia, Dirección General de Bellas Artes, Servicio Nacional de Excavaciones Arqueológicas.

Schubart, H., \& V. Pascual, 1966, «Datación por el Carbono 14 de los estratos con cerámica cardial de la Coveta del'Or", Archivo de Prehistoria Levantina XI, 45-51.

Simpson, G., 2000-01, «Remembering Frederick Zeuner and others at the Institute of Archaeology», Archaeology International 2000-01, 9-10. 
Siret, H., \& L. Siret, 1887, Les premiers ages du métal dans le Sud-Est de l'Espagne: résultats des fouilles faites par les auteurs de 1887 á 1887, Anvers; Louvain, Namur: Imprimerie de Charles Peeters; Impr. Paul Godenne.

Siret, H., \& L. Siret, 1890, Las primeras edades del metal en el sudeste de España, Barcelona: Imprenta de Henrich y Cia.

Siret, L., 1913, Questions de Chronologie et d'Ethnographie ibériques. Tome I: De la fin du Quaternaire a la fin du Bronze, Paris: Paul Geuthner.

Stika, H.-P., 2010, «In Memoriam Maria Hopf (1914-2008)», Madrider Mitteilungen 51: 555-558.

Uerpmann, H.-P., 1971, Die Tierknochenfunde aus der Talayot-Siedlung von S'Illot (San Lorenzo / Mallorca). Studien über frühe Tierknochenfunde von der Iberischen Halbinsel 2. München, Madrid: Institut für Palaeoanatomie, Domestikationsforschung und Geschichte der Tiermedizin der Universität München; DAI-Abteilung Madrid, 1986.

Varela, T., 1975a, "Análisis antropológico de los restos óseos de la necrópolis hispano-visigoda de Segóbriga (Cuenca)", en: M. Almagro Basch (ed.), La necrópolis hispano-visigoda de Segóbriga (Riba de Saelices, Cuenca), Madrid: Ministerio de Educación y Ciencia. Dirección General del Patrimonio Artístico y Cultural (Excavaciones Arqueológicas en España 84), 131-135.

Varela, T., 1975b, «Apéndice. Estudio antropológico de los restos óseos de Casa Herrera (Badajoz)», en: L. Caballero Zoreda, T. Ulbert (eds.), La basilica paleocristiana de Casa Herrera en las cercanias de Mérida (Badajoz) [con un apéndice redactado por Tito A. Varela], Madrid: Ministerio de Educación y Ciencia. Comisaría Nacional del Patrimonio Artístico. Dirección General del Patrimonio Artístico y Cultural, 237-248.

VILA, A., \& J. Estévez, 1989, “'Sola ante el peligro”: la arqueología ante las ciencias auxiliares», Archivo Español de Arqueología 62, 272-278.

Waterbolk, H. T., 1999, "Albert Egges van Giffen 1884-1973», en: T. Murray (ed.), Encyclopedia of Archaeology. The Great Archaeologists. vol 1, Santa Barbara: ABC-CLIO, 335-356.

Watson, P. J., S. A. Leblanc \& C. L. Redman, 1974, El método cientifico en arqueología [(1971) Explanation in Archaeology: An Explicitly Scientific Approach], Madrid: Alianza Universidad.

Zeuner, F. E., 1956, Geocronologia: la datación del pasado. Una introducción a la cronología prehistórica, Barcelona: Omega.

Zeuner, F. E., 1959, El periodo pleistoceno: su clima, cronología y sucesiones de la fauna, Madrid: Consejo Superior de Investigaciones Científicas. 